\title{
Spiders of the genus Haplodrassus (Araneae: Gnaphosidae) from Japan
}

\author{
Takahide Kamura \\ Biological Laboratory, Otemon Gakuin University, 2-1-15, Nishi-Ai, Ibaraki, \\ Osaka, 567-8502 Japan \\ E-mail: kamura@res.otemon.ac.jp
}

\begin{abstract}
Six species of the genus Haplodrassus (Gnaphosidae) are reported from Japan: H. pugnans (Simon 1880), H. cognatus (Westring 1861), H. kanenoi Kamura 1995, and three new species. It became clear that a Japanese species was incorrectly identified with Haplodrassus montanus Paik \& Sohn 1984, and this species is described as new to science under the name of $H$. hatsushibai. Furthermore, two new species are described under the names of $H$. mayumiae and $H$. nojimai.
\end{abstract}

Key words - Gnaphosidae, Haplodrassus, taxonomy, new species, Japan

\section{Introduction}

The first record of the gnaphosid genus Haplodrassus from Japan was made by Hayashi (1984) who identified specimens collected from Hokkaido with Haplodrassus signifer (C. L. Koch 1839). However, Yaginuma detected Hayashi's identification was incorrect (pers. com.), and treated the spider as H. pugnans (Simon 1880) in his book (Yaginuma 1986). Subsequently, Chikuni (1989) reported two Japanese species of this genus, $H$. pugnans and $H$. montanus Paik \& Sohn 1984. The latter species described from Korea was newly recorded from Japan. Furthermore, Kamura (1995) described H. kanenoi Kamura 1995, and reported H. cognatus (Westring 1861) as new to the Japanese fauna.

After examining specimens of this genus collected from various parts of Japan, I recognized that the spider previously identified with $H$. montanus in Japan is not true montanus, but an undescribed species, and found additional two species new to science. In this paper, I treat three known species and three new species of this genus from Japan.

The type specimens of the new species described in this paper are deposited in the collection of the Department of Zoology, National Science Museum, Tokyo (NSMT).

The abbreviations used in this paper are as follows: ALE, anterior lateral eye; AME, anterior median eye; MOA, median ocular area; p, proventral; PLE, posterior lateral eye; PME, posterior median eye; $r$, retroventral. Eye size means length of long axis of an eye, but measurement of posterior median eye was made at horizontal level. All measurements are given in $\mathrm{mm}$.

I would like to express my sincere thanks to Dr. Christine Rollard, Muséum National d'Histoire Naturelle, Paris, for loaning the type specimens of Drassus pugnans, and Dr. Norman I. Platnick, American Museum of Natural History,
New York, for loaning the specimens of Haplodrassus hiemalis. My thanks are also due to the following persons for their offering or loaning the specimens used in this study: Mr. Shingo Hatsushiba, Tokyo, Mr. Yoh Ihara, Hiroshima, Mr. Susumu Kaneno, Osaka, Mr. Ken-ichi Kumada, Mie, Ms. Mayumi Matsuda, Hokkaido, Mr. Koichi Nojima, Osaka, Dr. Hirotsugu Ono, National Science Museum, Tokyo, Dr. Akio Tanikawa, The University of Tokyo, Dr. Nobuo Tsurusaki, Tottori University, Mr. Tadakiyo Yamano, Osaka, Mr. Kazuyuki Yamamoto, Hyogo, and Mr. Nobuki Yasuda, Hokkaido.

Genus Haplodrassus Chamberlin 1922

Description. Anterior eye row slightly recurved and posterior eye row slightly procurved as seen from above. PME separated from each other usually by less than half of the eye size, and further apart from PLE. Chelicera with three (rarely two) teeth on promargin of fang furrow, and two teeth on retromargin. Trochanters of legs without ventral notch. Male abdomen with a dorsal scutum rather small, often indistinct. Female posterior median spinneret somewhat swollen proximally (Fig. 6). Male palp with embolus thick, median apophysis hook-like, terminal apophysis developed, and retrolateral tibial apophysis flattened. Epigynum with an anterior hood and lateral margins.

Haplodrassus pugnans (Simon 1880)

(Figs. 1-6)

Drassus pugnans Simon 1880, p. 118, pl. 3, figs. 20-21.

Haplodrassus signifer: Hayashi 1984, p. 13, figs. 12-26 (misidentification).

Haplodrassus pugnans: Yaginuma 1986, p. 193, fig. 108 (1); Matsuda 1987, p. 23, figs. 23-26; Chikuni 1989, pp. 121 (fig. 16) \& 251 . 
For other literature see Platnick (2006).

Specimens examined. 2ㅈ1우1우 juv. (syntypes), Peking, China, deposited in Muséum National d'Histoire Naturelle, Paris (B. 613, No. 363); 10, Higashioribe, Kamishihorocho, Kato-gun, Hokkaido, Japan, 7.V.1985, M. Matsuda leg.; 1우, 18.V.1987, same locality and collector; 1주우, Gokashô (riverside of Echi-gawa River, altitude $120 \mathrm{~m}$ ), Higashiomi-shi, Shiga Pref., Japan, 15.IV.1994, S. Kaneno leg. (1우 matured after breeding); $1 \delta^{\pi}$, Shirakawadai, Sumaku, Kobe-shi, Hyogo Pref., Japan, 22.II.1979, T. Yamano leg.; $1 \delta^{\Uparrow} 1$ 우, Tottori-Sakyu, Tottori-shi, Tottori Pref., Japan, 21.IV.1991, N. Tsurusaki et al. leg.; 1우, Ôkushi, Ôsakikamijima-cho, Toyota-gun, Hiroshima Pref., Japan, 6.V.1993, Y. Ihara leg.

Measurements. Body length $\sigma^{3} 4.05-6.18$, 우5.58-8.90. Carapace length $\delta^{\top} 1.70^{-3.34}$, 우2.38-3.10; width $\delta^{\Uparrow} 1.25^{-}$ 2.41, 우1.80-2.30. Abdomen length ${ }^{\rtimes} 2.25-3.18$, 우3.10-
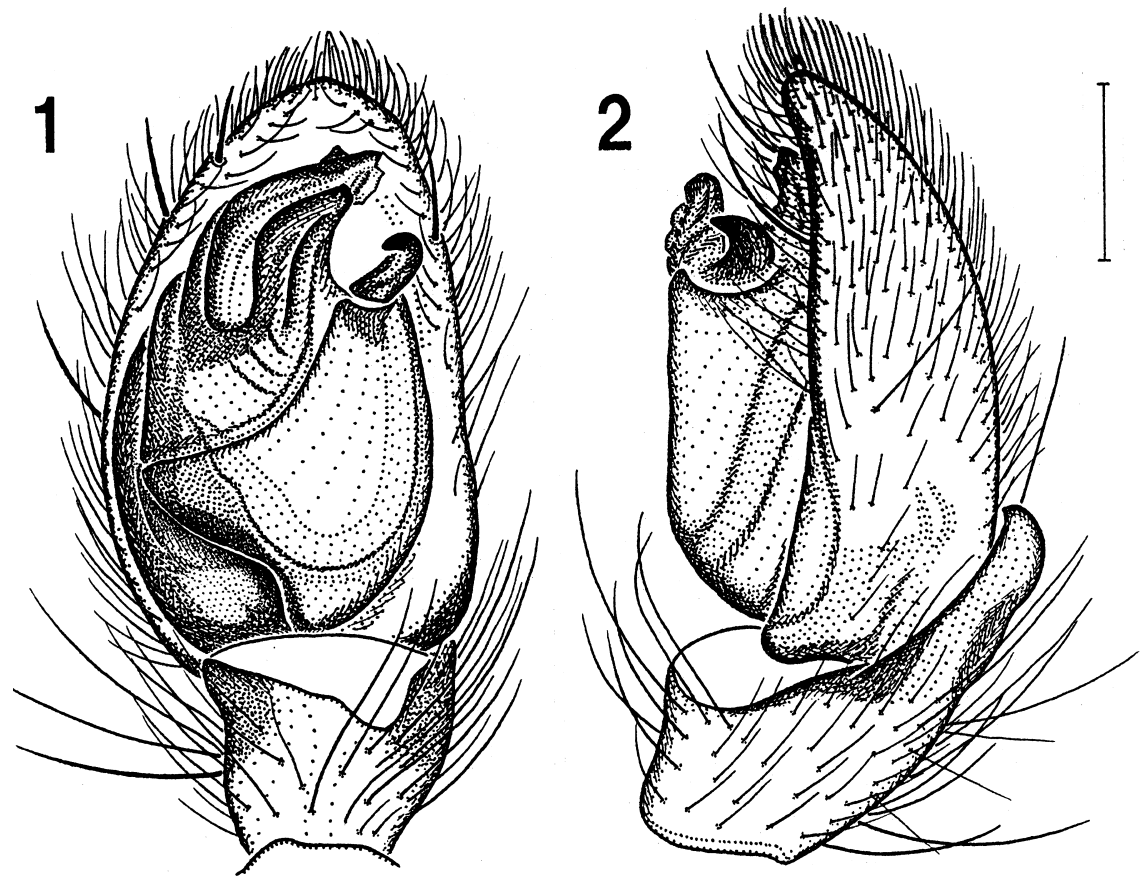

4

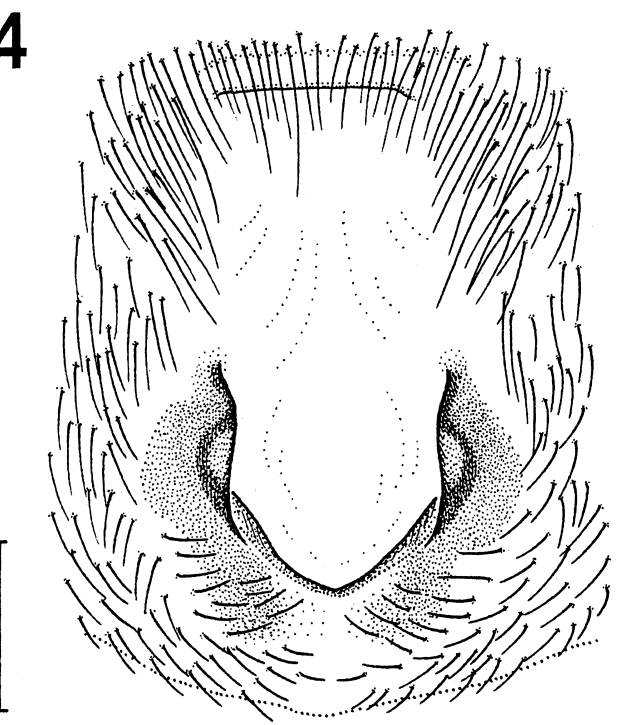

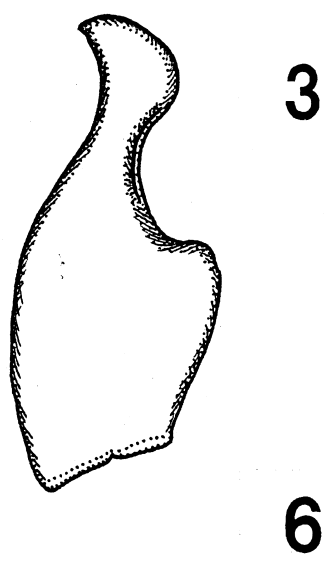

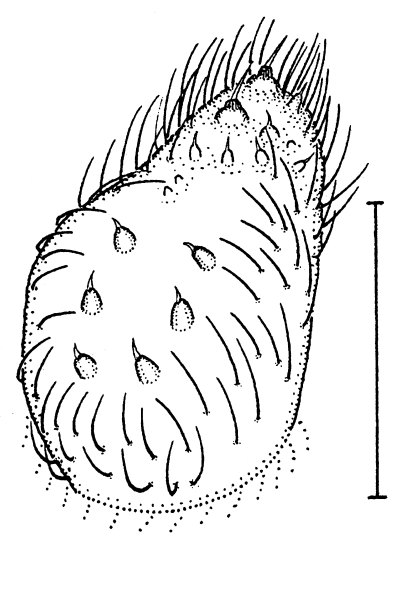

5
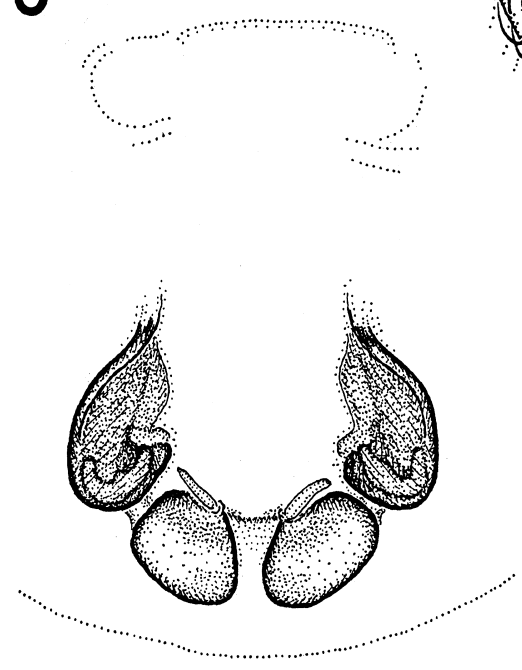

Figs. 1-6. Haplodrassus pugnans (Simon 1880) (1-3, Hyogo Pref.; 4-6, Tottori Pref.) - 1, Left male palp, ventral view; 2, same, retrolateral view; 3 , tibia of male palp, dorsal view; 4, epigynum, ventral view; 5 , female genitalia, dorsal view; 6 , left female posterior median spinneret, posterodorsal view. (Scales: $0.2 \mathrm{~mm}$ ) 
5.80; width $\overbrace{}^{\Im} 1.25^{-1.85}$, 우 $1.85^{-3}-43$.

Ventral spines on legs I and II. $\delta^{\top}$ : Tibiae: I and II $0^{-0} 0^{-0}$; metatarsi: I $0-0-0$, II $1 \mathrm{p}^{-} 0^{-0}$ or $2^{-}-0-0$. ㅇ: Tibiae: I and II $0-0-0$; metatarsi: I $0-0-0$, II $2-0-0$.

Male palp (Figs. 1-3): terminal apophysis narrow; retrolateral tibial apophysis widened apically. Epigynum and female genitalia as shown in Figs. 4-5.

Distribution. Japan (Hokkaido, Honshu); China to North Africa (Platnick, 2006).

\section{Haplodrassus cognatus (Westring 1861)}

(Figs. 7-8)

Drassus cognatus Westring 1861, p. 343.

Haplodrassus cognatus: Kamura 1995, p. 126, figs. 7-8.

For other literature see Platnick (2006).

Specimens examined other than 1 우 shown in Kamura (1995). 1우, Shibetsu-shi, Hokkaido, Japan, 22.V.1963, T.

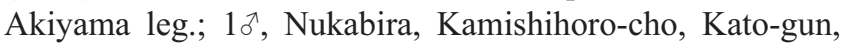
Hokkaido, Japan, 26.VI-4.VII.1995, M. Matsuda leg.

Measurements. $10^{\text {}}$ from Kamishihoro-cho: Body length 7.65; carapace length 3.55 , width 2.75 ; abdomen length 4.10, width 2.25. 1 ㅇ from Shibetsu-shi: Body length 8.80; carapace length 4.20 ; width 3.10 ; abdomen length 4.60 , width 2.90 .

Ventral spines of legs I \& II. $0^{7}$ : Tibiae: I $0-0-2$, II $0-1 p^{-}$ 2 or $0-2-2$; metatarsi: I and II $2-0-0$. ㅇ: Tibiae: I and II $0^{-}$ $0^{-} 0$ or $0^{-} 0^{-1} \mathrm{p}$; metatarsi: I $1 \mathrm{p}^{-} 0^{-} 0$ or $2^{-} 0^{-} 0$, II $2^{-} 0^{-} 0$.

Male palp (Figs. 7-8): terminal apophysis large, with a pointed projection on prolateral side. Description of female was made by Kamura (1995).

Distribution. Japan (Hokkaido); the Palearctic region (Platnick 2006).

\section{Haplodrassus kanenoi Kamura 1995}

(Figs. 19-20)

Haplodrassus kanenoi Kamura 1995, p. 123, fig. 1-6.

Specimens examined other than those listed in Kamura (1995). 1우, riverside of Sonose-gawa River, Tokushima-shi, Tokushima Pref., Japan, 18.VII.2002, collector unknown.

Measurements. 1우 from Tokushima Pref.: Body length 6.23 ; carapace length 2.58 , width 1.75 ; abdomen length 3.65 , width 2.10 .

Ventral spines on legs I and II. ${ }^{\top}$ : Tibiae: I and II $0^{-0} 0$; metatarsi: I $0-0-0$, II $2-0-0$. ㅇ: Tibiae: I and II $0-0-0$; metatarsi: I $0^{-} 0^{-0}$, II $0^{-} 0^{-} 0,1 \mathrm{r}^{-} 0^{-} 0$ or $2^{-} 0^{-} 0$.

Description was made by Kamura (1995). Diagnostic features in male palp: embolus with a small translucent projection beside embolar tip and a pointed projection rather small on dorsal side (Figs. 19-20). The pointed projection was incorrectly regarded as embolar tip in Kamura (1995).

Distribution. Japan (Honshu, Shikoku).
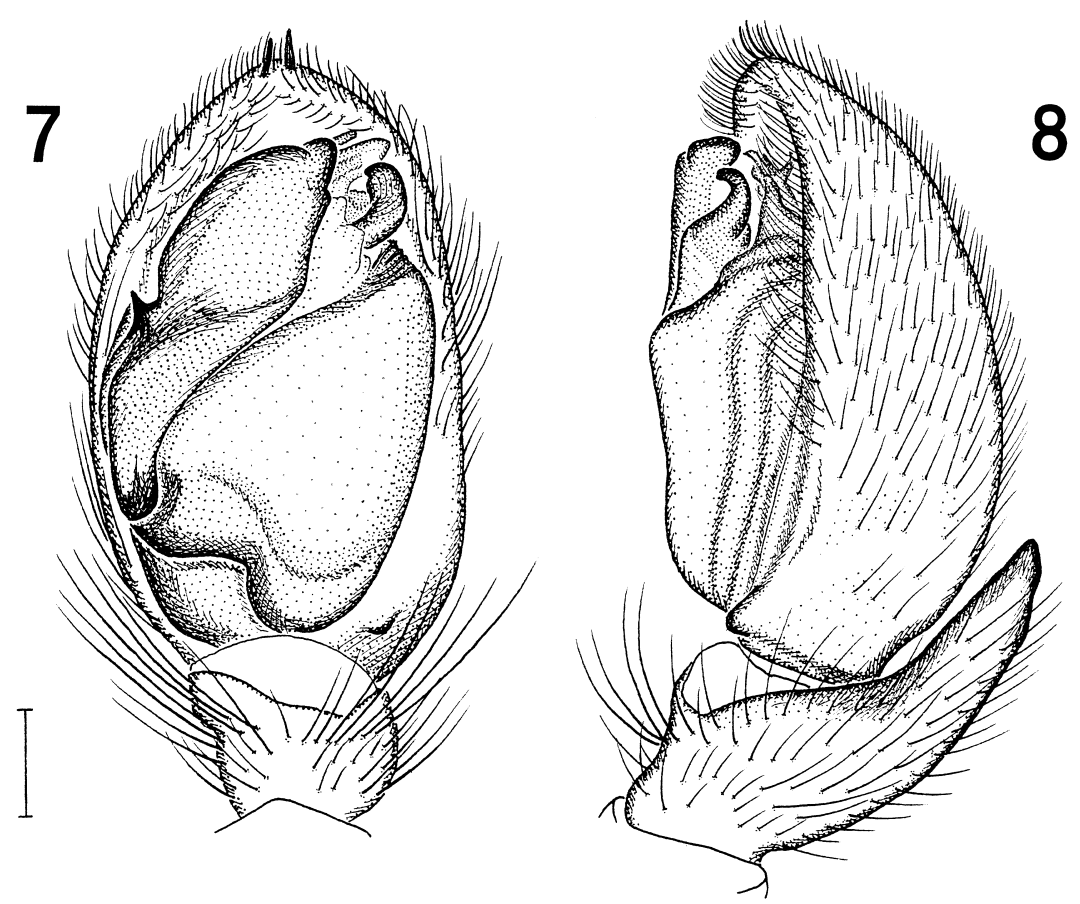

Figs. 7-8. Haplodrassus cognatus (Westring 1861) (Hokkaido) - 7, Left male palp, ventral view; 8, same, retrolateral view. (Scale: 0.2 $\mathrm{mm})$ 

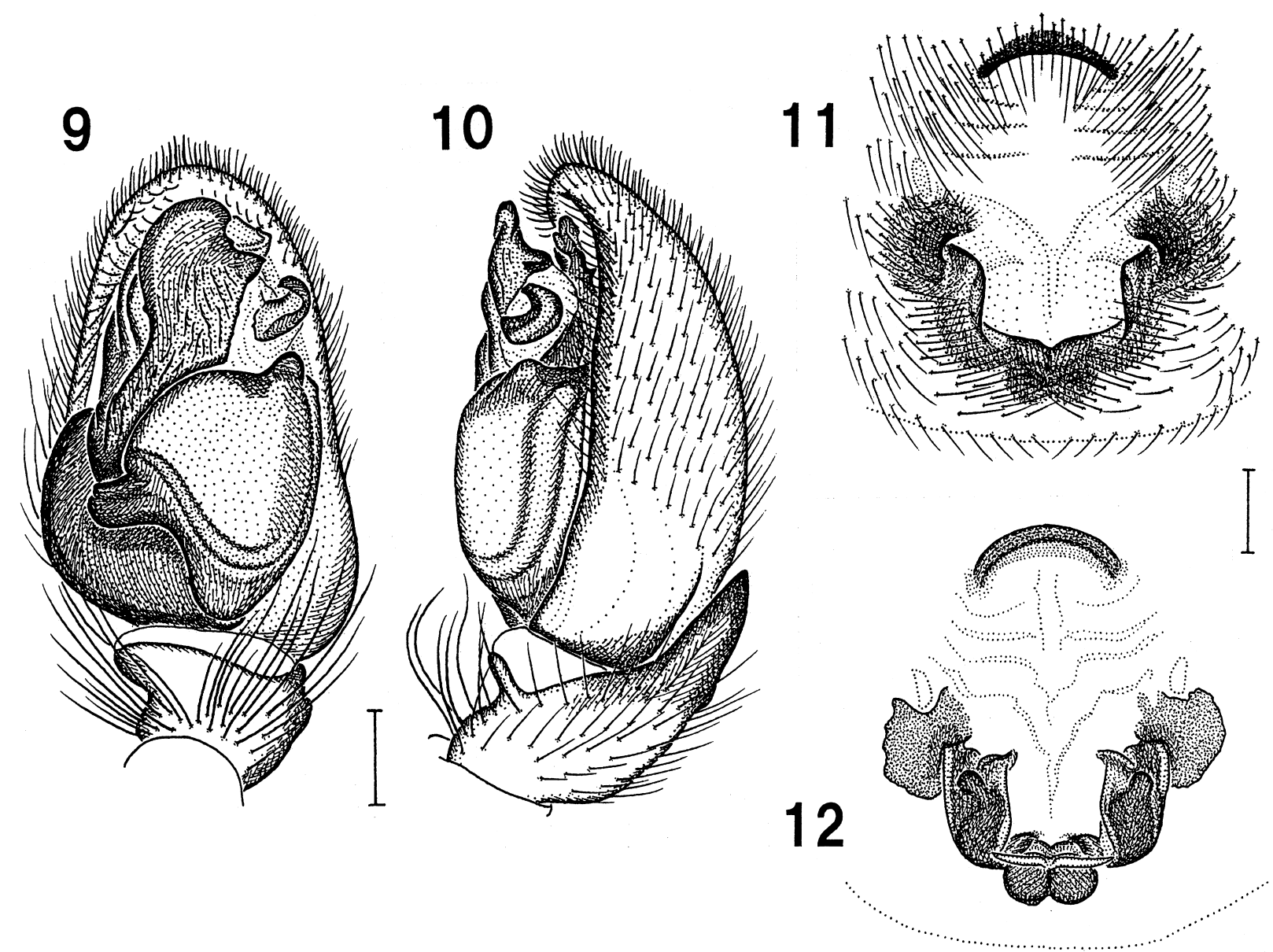

Figs. 9-12. Haplodrassus mayumiae n. sp. (9-10, holotype; 11-12, paratype) - 9, Left male palp, ventral view; 10, same, retrolateral view; 11, epigynum, ventral view; 12, female genitalia, dorsal view. (Scales: $0.2 \mathrm{~mm}$ )

Haplodrassus mayumiae $\mathrm{n}$. sp.

[Japanese name: Tokachi-haitakagumo] (Figs. 9-12, 17-18)

Type series. Holotype: $\delta^{\lambda}$, near mouth of Tokachi-gawa River, Toyokoro-cho, Nakagawa-gun, Hokkaido, Japan, 927.VI.1993, K. Shibata leg. (NSMT-Ar 5951). Paratypes: $10^{\top} 1$ 우, same data as for the holotype (10: NSMT-Ar 5952; 1우: NSMT-Ar 5953).

Other specimens examined. $2 \sigma^{7}$, same data as for the holotype; 1ð, Sarobetsu, Toyotomi-cho, Teshio-gun, Hokkaido, Japan, 9.VI.1991, N. Yasuda leg. (NSMT-Ar 5954); $10^{\lambda}$, 11.VII.1991, same locality and collector; $10^{\lambda}$, riverside of Abukuma-gawa River, Miyagi Pref. or Fukushima Pref., Japan (detail locality unknown), 29.V.1997, collector unknown.

Specimens examined for comparison. Haplodrassus hiemalis (Emerton 1909): 10, Ramsey, New Jersey, USA, 3.VI.1934, Gertsch leg., deposited in American Museum of Natural History (AMNH); 1 우, Port Credit (latitude $43^{\circ} 33^{\prime} \mathrm{N}$, longitude $79^{\circ} 35^{\prime}$ W), Ontario, USA, 15.VI.1946, S. Harrod leg., deposited in AMNH.

Diagnosis. This new species resembles H. kanenoi Kamura 1995 and H. hiemalis (Emerton 1909), especially in whole structure of male palp these species are very similar one another (Figs. 9-10 \& 13-14; Kamura 1995, figs. 1-2). Diagnostic features are as follows. Male: $H$. kanenoi has a translucent small projection beside embolar tip (Figs. 1920 ), while such projection is not found in this new species (Figs. 17-18); a projection situated on dorsal side of embolus is smaller in this new species (Figs. 17-18) than in $H$.

Table 1. Measurements of legs of Haplodrassus mayumiae n. sp. (male holotype/female paratype)

\begin{tabular}{ccccccc}
\hline Leg & Femur & Patella & Tibia & Metatarsus & Tarsus & Total \\
\hline I & $2.05 / 2.05$ & $1.23 / 1.30$ & $1.70 / 1.68$ & $1.34 / 1.33$ & $1.01 / 0.90$ & $7.33 / 7.26$ \\
II & $1.73 / 1.73$ & $1.00 / 1.08$ & $1.28 / 1.29$ & $1.11 / 1.08$ & $0.86 / 0.79$ & $5.98 / 5.97$ \\
III & $1.45 / 1.48$ & $0.78 / 0.79$ & $0.98 / 1.00$ & $0.99 / 0.98$ & $0.73 / 0.66$ & $4.93 / 4.91$ \\
IV & $2.13 / 2.15$ & $1.05 / 1.13$ & $1.75 / 1.78$ & $1.73 / 1.76$ & $0.88 / 0.83$ & $7.54 / 7.65$ \\
\hline
\end{tabular}




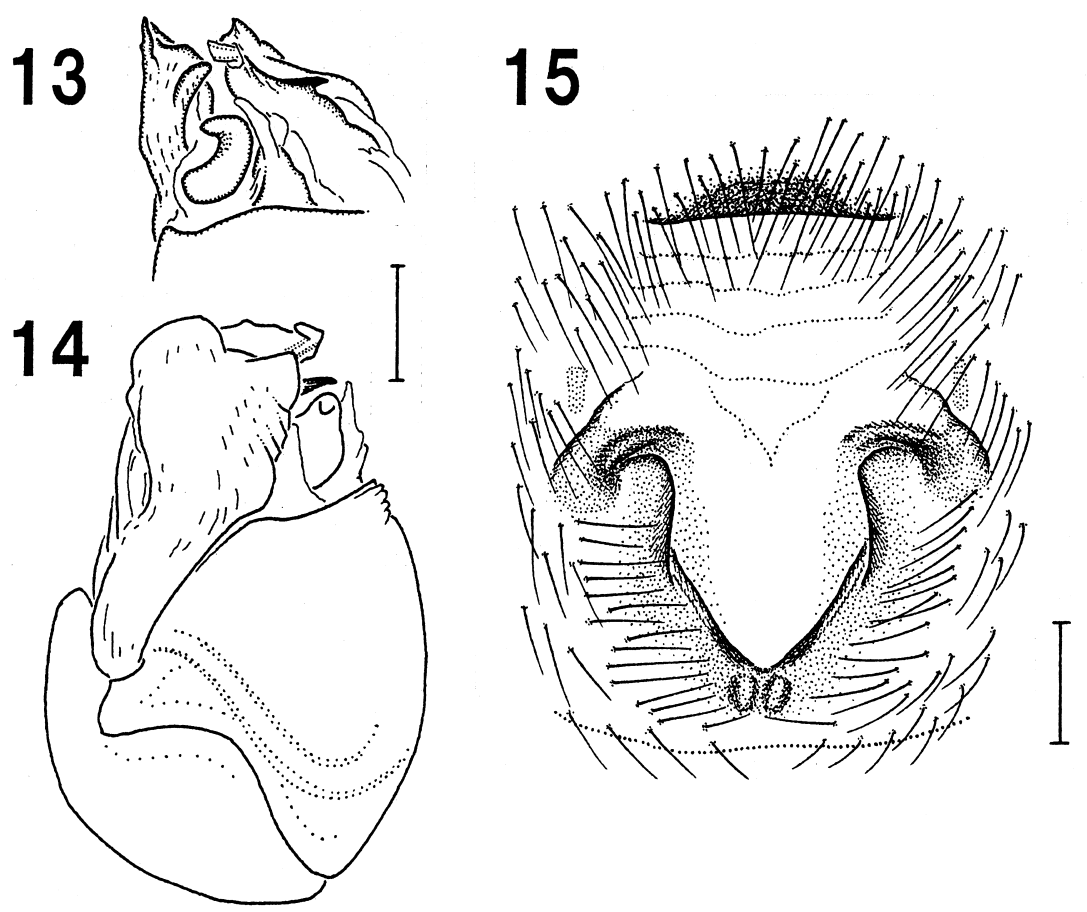

16

Figs. 13-16. Haplodrassus hiemalis (Emerton 1909) (13-14, New Jersey, USA; 15-16, Ontario, USA) — 13, Apical part of left male palp, retrolateral view; 14, left male palp (bulb), ventral view; 15, epigynum, ventral view; 16, female genitalia, dorsal view. (Scales: 0.2 $\mathrm{mm}$ )
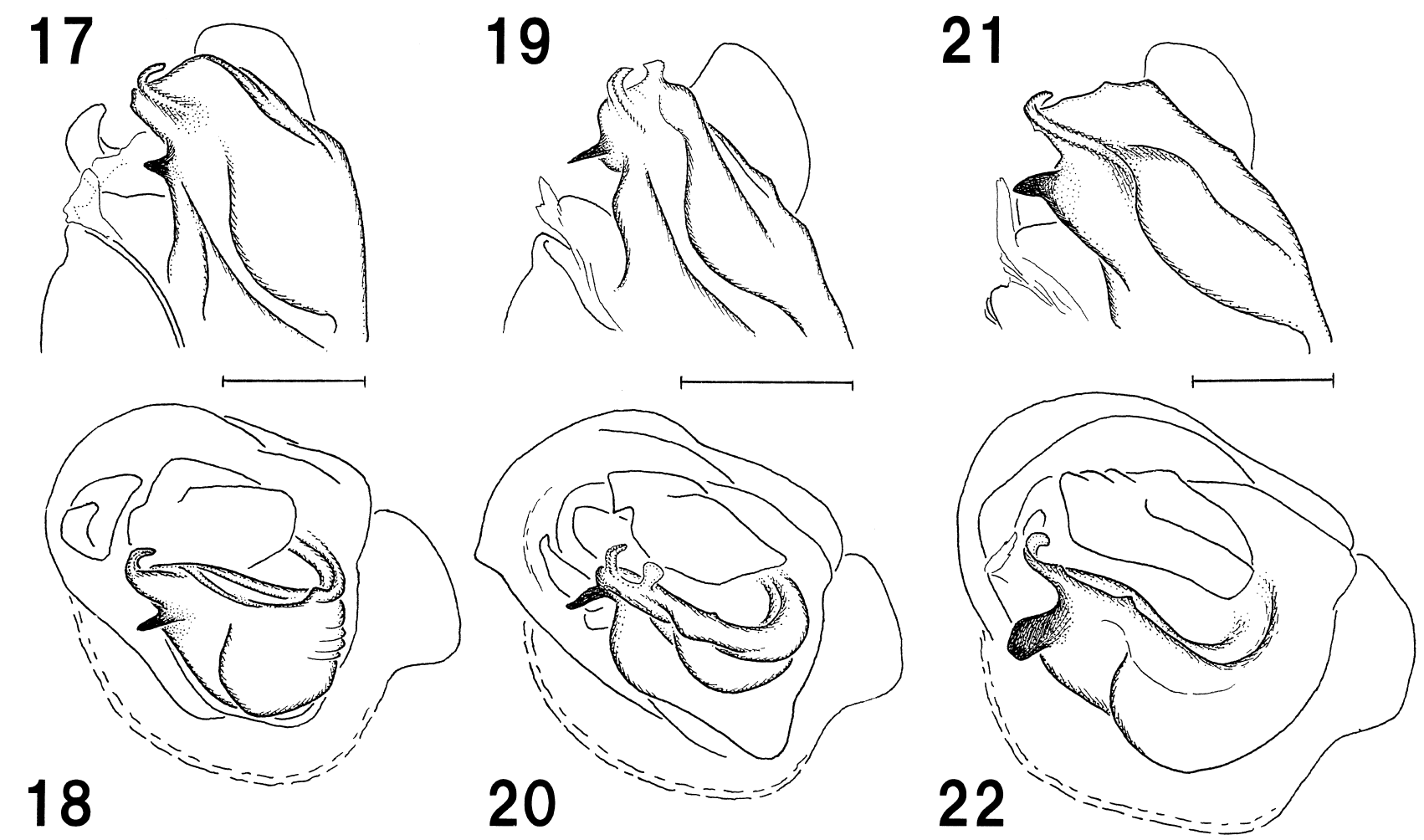

Figs. 17-22. Apical parts of left male palps (17, 19 \& 21, dorsal view; 18, 20 \& 22, apical view) - 17-18, Haplodrassus mayumiae n. sp. (paratype); 19-20, H. kanenoi Kamura 1995 (Chiba Pref.); 21-22, H. hiemalis (Emerton 1909) (New Jersey, USA). (Scales: 0.2 mm) 
hiemalis (Figs. 21-22). Female: in this new species portion between lateral margins of epigynum is shorter (Fig. 11) than in the other two species (Fig. 15; Kamura 1995, fig. 5).

Description. Measurements based on the male holotype and the female paratype. Body length ${ }^{\top} 6.10$, +6.53 . Carapace length $\delta^{3} 2.65$, 우2.83; width 32.00 , + 2.15 . Abdomen length 33.45 , 우 3.70 ; width $\$ 2.15$, 우 2.25 . Eye sizes: AME $\delta^{\top} 0.16$, 우0.16; ALE $\delta^{\top} 0.18$, 우0.16; PME ${ }^{\top} 0.16$, 우 0.17 ; PLE $\delta^{\curlyvee} 0.13$, 우 0.14 . Distances between eyes: AME-

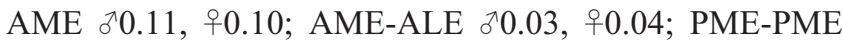
${ }^{\top} 0.05$, 웅.04; PME-PLE $\overbrace{}^{\top} 0.17$, 우 0.16 ; ALE-PLE $\delta^{\top} 0.08$, + 0.09 . MOA anterior width $\delta^{\top} 0.40$, 우 0.41 ; posterior width ${ }^{\top} 0.37$, o 0.38 ; length $\delta^{\top} 0.41$, + 0.44 . Clypeus height $\delta^{\top} 0.13$, +o.14. Length of legs as in Table 1.

Variation. $0^{7}$ : Body length 5.78-6.65; carapace length 2.55-2.95, width 1.95-2.25; abdomen length 3.13-3.85, width $1.75-2.30$.

Ventral spines on legs I and II. ${ }^{\top}$ : Tibiae: I and II $0^{-0}-0$; metatarsi: I $0^{-} 0^{-} 0$, II $1 \mathrm{r}^{-} 0^{-} 0$ or $2^{-} 0^{-} 0$. 우: Tibiae: I and II $0^{-}$ $0-0$; metatarsi: I $0^{-} 0-0$, II $2^{-}-0$.

Male palp (Figs. 9-10, 17-18): terminal apophysis with apical part rounded; embolar base with a pointed projection rather small on dorsal side; embolar tip bent. Epigynum (Fig. 11): portion between lateral margins wider than long. Female genitalia with epigynal ducts massive (Fig. 12).

Color. Cephalothorax and appendages yellowish brown to light reddish brown, mouth parts and sternum somewhat darker. Abdomen grayish brown.

Distribution. Japan (Hokkaido, Honshu).

Etymology. The specific name is dedicated to Ms. Mayumi Matsuda, Hokkaido.
Haplodrassus hatsushibai n. sp.

[Japanese name: Musashi-haitakagumo]

(Figs. 23-31)

Haplodrassus montanus: Chikuni 1989, pp. 121 (fig. 17) \& 251 (misidentification).

Type series. Holotype: 우, Otaka-no-mori (latitude $35^{\circ}$ $41^{\prime} 02^{\prime \prime} \mathrm{N}$, longitude $139^{\circ} 26^{\prime} 18^{\prime \prime} \mathrm{E}$, altitude $69 \mathrm{~m}$ ), Yaho, Kunitachi-shi, Tokyo, Japan, 13.IV.2006, S. Hatsushiba leg. (NSMT-Ar 5955). Paratypes: $1{ }^{\widehat{ }}$, same locality and collector as for the holotype, 18.IV.2006 (NSMT-Ar 5956); 1우, same locality as for the holotype, 18.IV.2006, T. Horiguchi leg. (NSMT-Ar 5957).

Other specimens examined. 1ㅇ, 13.IV.2006, same locality and collector as for the holotype; 1우, Hirai, Hinodemachi, Nishitama-gun, Tokyo, Japan, 29.VII.1981, Y. Kunimi leg.; 10 $0^{\lambda}$, northeastern coast of Lake Horokayantounuma, Taiki-cho, Hiroo-gun, Hokkaido, Japan, 9-27.VI. 1993, K. Shibata leg.

Diagnosis. This new species is similar to $H$. dentatus $\mathrm{Xu}$ \& Song 1987 described from China, but is distinguished from the latter by having a pointed projection on terminal apophysis in male palp (Fig. 25; cf. Xu and Song 1987, fig. 1; Song et al. 2004, fig. 77C), and a pair of auriform lobes on lateral parts of epigynum (Fig. 28) and epigynal ducts expanded posterolaterally (Fig. 29).

This new species also resembles $H$. montanus Paik \& Sohn 1984 described from Korea. Female of this new species is separated from that of the latter by the auriform lobes in epigynum and posterolaterally expanded epigynal ducts stated above (Figs. 28-29; cf. Paik \& Sohn 1984, figs. 19-

Table 2. Measurements of legs of Haplodrassus hatsushibai n. sp. (male paratype/female holotype)

\begin{tabular}{ccccccc}
\hline Leg & Femur & Patella & Tibia & Metatarsus & Tarsus & Total \\
\hline I & $1.43 / 1.33$ & $0.83 / 0.80$ & $1.10 / 0.94$ & $0.89 / 0.76$ & $0.71 / 0.66$ & $4.96 / 4.49$ \\
II & $1.20 / 1.13$ & $0.73 / 0.70$ & $0.83 / 0.76$ & $0.76 / 0.66$ & $0.63 / 0.60$ & $4.15 / 3.85$ \\
III & $1.05 / 0.98$ & $0.58 / 0.55$ & $0.65 / 0.60$ & $0.79 / 0.71$ & $0.54 / 0.53$ & $3.61 / 3.37$ \\
IV & $1.45 / 1.41$ & $0.78 / 0.78$ & $1.11 / 1.09$ & $1.24 / 1.15$ & $0.68 / 0.70$ & $5.26 / 5.13$ \\
\hline
\end{tabular}
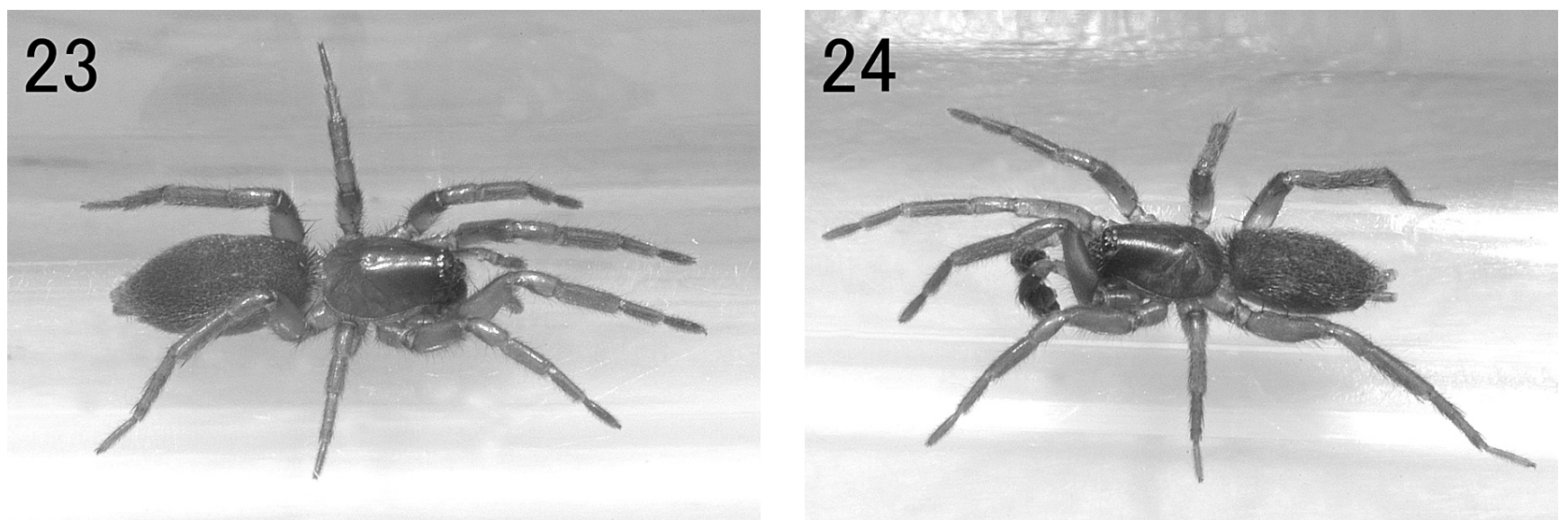

Figs. 23-24. Haplodrassus hatsushibai n. sp. - 23, Female paratype; 24, male paratype. 

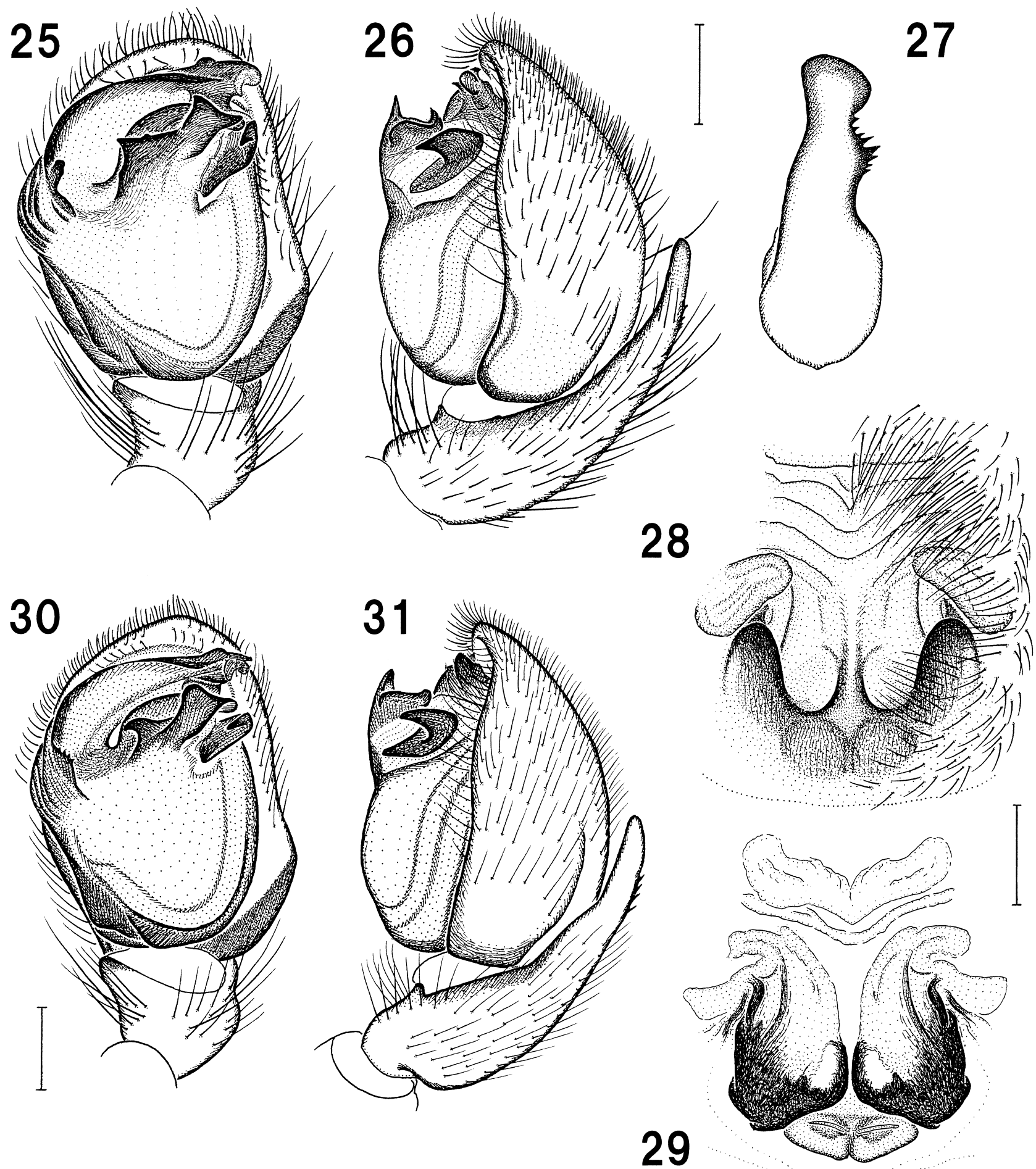

Figs. 25-31. Haplodrassus hatsushibai n. sp. (25-27, paratype; 28-29, holotype; 30-31, Hokkaido) - 25 \& 30, Left male palps, ventral views; 26 \& 31, same, retrolateral views; 27, tibia of left male palp, dorsal view; 28, epigynum, ventral view; 29, female genitalia, dorsal view. (Scales: $0.2 \mathrm{~mm}$ )

21). Males of these species are very similar to each other in palpal structure (Figs. 25-27; cf. Paik \& Sohn 1984, figs. $15^{-16} \& 22$ ) except pointed projection slightly larger on terminal apophysis in this new species.

Description. Measurements based on the male paratype and the female holotype. Body length $\varangle 4.23$, + 4.43 . Carapace length $\delta^{\top} 2.00$, 우1.90; width $\delta^{\top} 1.58$, 우1.38.

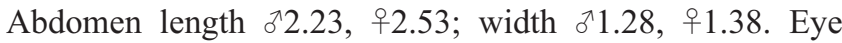

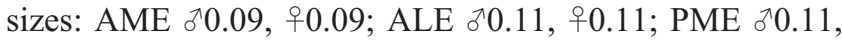
우 0.11; PLE $\jmath^{\Im} 0.07$, 우0.09. Distances between eyes: AME- 
AME ${ }^{\top} 0.05$, 우0.07; AME-ALE ${ }^{\top} 0.01$, 우0.01; PME-PME ð0.03, 우0.03; PME-PLE ð00.07, 우0.07; ALE-PLE ð 0.05 , 우 0.05 . MOA anterior width $\delta^{\top} 0.23$, 우 0.24 ; posterior width $\jmath^{\top} 0.24$, ㅇ 0.24 ; length ${ }^{\top} 0.27$, + 0.28 . Clypeus height $\gtrsim 0.11$, ㅇ․09. Length of legs as in Table 2 .

Variation. 우 Body length 4.43-5.30; carapace length 1.90-2.40, width 1.38-1.85; abdomen length 2.53-2.90; width 1.38-1.75. 10 from Hokkaido: Body length 5.10; carapace length 2.15, width 1.75; abdomen length 2.95, width 1.85

Ventral spines on legs I and II. ऽ$^{-}$Tibiae: I and II $0^{-} 0^{-} 0$; metatarsi: I $0^{-} 0^{-} 0$ or $2^{-} 0^{-} 0$, II $2^{-} 0^{-} 0$. 우: Tibiae: I and II $0^{-}$ $0-0$; metatarsi: I and II $2-0-0$.

Male palp (Figs. 25-27): tegulum massive with some fine ridges on prolateral side; terminal apophysis with a projection protruding prolaterally; tibial apophysis with distinct serration on prolateral side. Epigynum with a longitudinal ridge on posterior part, a pair of auriform lobes on lateral parts, and copulatory openings situated on posterolateral parts (Fig. 28); copulatory openings covered with mating plugs in the female paratype. Female genitalia with epigynal ducts wide (Fig. 29).

Color. Cephalothorax brown; eye area, mouth parts and sternum darker. Legs light brown to brown. Abdomen dark grayish brown.

Remarks. Although this new species and $H$. montanus are very similar to each other in the male palpal structure as stated above, they greatly differ in the structure of epigynum and female genitalia.

One male examined from Hokkaido is slightly different from the male paratype from Tokyo in palpal structure (Figs 25-26 \& 30-31). Although it is difficult to judge whether they belong to same species, I regarded them as intraspecific and geographical variation in this study.

Distribution. Japan (Hokkaido, Honshu).

Etymology. The specific name is dedicated to $\mathrm{Mr}$. Shingo Hatsushiba, Tokyo.

Haplodrassus nojimai n. sp.

[Japanese name: Houki-haitakagumo]

(Figs. 32-33)

Type series. Holotype: 우, Saigasaki (riverside of Tenjingawa River), Kurayoshi-shi, Tottori Pref., Japan, 30.VII.1996, K. Nojima leg. (NSMT-Ar 5958). Paratype: 1우, Higashi-shindenba, Hokuei-cho, Touhaku-gun, Tottori Pref., Japan, 17.VII.2001, K. Nojima leg. (NSMT-Ar 5959).

Diagnosis. This new species is easily distinguished from the other species of the genus by having inverted V-shaped margin situated on median part of epigynum (Fig. 32).

Description. 우. Measurements based on the female holotype. Body length 4.90. Carapace length 2.15; width 1.60. Abdomen length 2.75; width 1.85. Eye sizes: AME 0.10; ALE 0.11; PME 0.12; PLE 0.09. Distances between eyes: AME-AME 0.07; AME-ALE 0.01; PME-PME 0.03; PME-PLE 0.08; ALE-PLE 0.07. MOA anterior width 0.26;

Table 3. Measurements of legs of Haplodrassus nojimai n. sp. (female holotype)

\begin{tabular}{ccccccc}
\hline Leg & Femur & Patella & Tibia & Metatarsus & Tarsus & Total \\
\hline I & 1.46 & 0.90 & 1.02 & 0.84 & 0.70 & 4.92 \\
II & 1.24 & 0.76 & 0.82 & 0.76 & 0.66 & 4.24 \\
III & 1.10 & 0.63 & 0.64 & 0.80 & 0.58 & 3.75 \\
IV & 1.56 & 0.86 & 1.16 & 1.26 & 0.74 & 5.58 \\
\hline
\end{tabular}
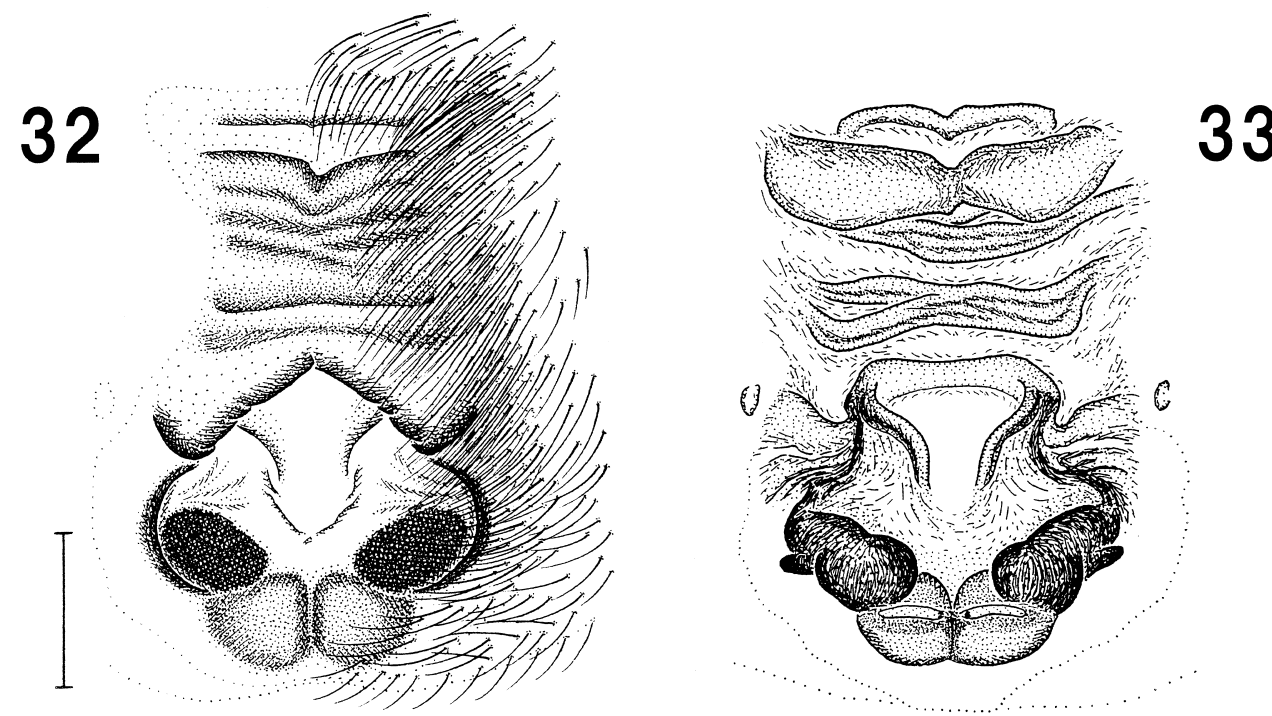

Figs. 32-33. Haplodrassus nojimai $\mathrm{n}$. sp. (holotype) - 32, Epigynum, ventral view; 33, female genitalia, dorsal view. (Scale: $0.2 \mathrm{~mm}$ ) 
posterior width 0.27 ; length 0.28 . Clypeus height 0.10 . Length of legs as in Table 3.

Variation. 1우 (paratype): Body length 4.05; carapace length 1.80, width 1.33; abdomen length 2.25, width 1.43 .

Ventral spines on legs I and II. ㅇ: Tibiae: I and II $0^{-} 0^{-} 0$; metatarsi: I $0^{-} 0^{-} 0$ or $1 \mathrm{p}^{-} 0^{-} 0$, II $2^{-} 0^{-}-0$.

Epigynum (Fig. 32): a pair of lateral margins relatively short, rounded; inverted V-shaped margin situated on median part; copulatory openings covered with mating plugs in the specimens examined. Female genitalia as shown in Fig. 33.

Color. Cephalothorax light reddish brown; eye area, mouth parts and sternum darker. Legs yellowish brown to light reddish brown. Abdomen grayish brown.

Male. Unknown.

Distribution. Japan (Honshu).

Etymology. The specific name is dedicated to $\mathrm{Mr}$. Koichi Nojima, Osaka.

\section{References}

Chamberlin, R. V. 1922. The North American spiders of the family Gnaphosidae. Proc. Biol. Soc. Wash. 35: 145-172.

Chikuni, Y. 1989. Pictorial Encyclopedia of Spiders in Japan. Kaiseisha, Tokyo, 308 pp. (In Japanese)

Emerton, J. H. 1909. Supplement to the New England spiders. Trans. Connecticut Acad. Arts Sci., 14: 171-236, pls. 1-12.
Hayashi, T. 1984. Drassodes lapidosus (Walckenaer, 1802) and Haplodrassus signifer (C. L. Koch, 1839). Atypus, 85: 9-18. (In Japanese)

Kamura, T. 1995. A new species and a newly recorded species of the genus Haplodrassus (Araneae: Gnaphosidae) from Japan. Acta Arachnol., 44: 123-127.

Koch, C. L. 1839. Die Arachniden, 6. Nürnberg, 156 pp.

Matsuda, M. 1987. Spiders of the Tokachi plain, Hokkaido, Japan (1). Bull. Higashi Taisetsu Mus. Nat. Hist., 9: 15-33. (In Japanese)

Paik, K. Y. \& Sohn, S. R. 1984. The Korean spiders of the genus Haplodrassus Chamberlin, 1922 (Araneae: Gnaphosidae). J. Inst. Nat. Sci., 3: 105-112.

Platnick, N. I. 2006. The World Spider Catalog, Version 7.0. Available from Internet: < http: //research.amnh.org/entomology/ spiders/catalog/INTRO1.html $>$.

Simon, E. 1880. Etudes arachnologiques. 11e Mémoire. XVII. Arachnides recueillis aux environs de Pékin par M. V. Collin de Plancy. Ann. Soc. Ent. France, Ser. 5, 10: 97-128, pl. 3.

Song, D. X., Zhu, M. S. \& Zhang, F. 2004. Fauna Sinica: Invertebrata Vol. 39: Arachnida: Araneae: Gnaphosidae. Science Press, Beijing, ix +362 pp.

Westring, N. 1861. Araneae svecieae. Göteborgs Kongl. Vet. Handl. 7: $1-615$.

Xu, Y. J. \& Song, D. X. 1987. A new species of the genus Haplodrassus (Araneae, Gnaphosidae) from China. J. Anhui Normal Univ. 1987(4): 83-85.

Yaginuma, T. 1986. Spiders of Japan in Color, new ed. Hoikusha, Osaka, xxiv +305 pp., 64 pls. (In Japanese)

Received September 25, 2006 / Accepted December 16, 2006 